\title{
Short-Term Prosocial Video Game Exposure Influences Attentional Bias Toward Prosocial Stimuli
}

\author{
Boyu Qiu, MS, ${ }^{1,2}$ Shuangju Zhen, PhD, ${ }^{1,2}$ Cui Zhou, BS, ${ }^{1,2}$ Jianping $\mathrm{Hu}, \mathrm{PhD},{ }^{3}$ and Wei Zhang, $\mathrm{PhD}^{1,2}$
}

\begin{abstract}
Research has shown that violent video games induce attentional bias toward aggressive information. However, the effects of prosocial video games on selective attention are poorly understood. This study investigated attentional bias toward prosocial stimuli at different presentation durations (i.e., 100, 500, and 1,250 milliseconds [ms]) after short-term prosocial video game exposure. Sixty males (mean age: 20.26 years; range: 19-23 years) participated in this study. Half of them played a prosocial video game for 30 minutes, whereas others played a neutral one. A spatial cueing paradigm was then used to investigate attentional bias. Results showed that there was both attention orientation and difficulty in attention disengagement toward prosocial stimuli when the presentation lasted $100 \mathrm{~ms}$ in the prosocial game group, but not in the neutral group. There was no group difference at 500 or $1,250 \mathrm{~ms}$, suggesting that the attentional bias toward prosocial information might occur at the early stages of cognitive processing. These results provided initial evidence of the influence of prosocial video games on cognitive processing and advanced our understanding of related theories.
\end{abstract}

Keywords: prosocial video games, attentional bias, spatial cueing paradigm

\section{Introduction}

A S VIDEO GAMEs become extremely popular, ${ }^{1,2}$ the potential effects of video games have gradually caught the attention of researchers. Given that most popular video games contain violent components, ${ }^{3,4}$ previous studies mainly focused on violent video games. These studies found that violent games enhanced aggressive thoughts and emotions, and further resulted in aggressive behaviors. ${ }^{5-7}$ Recently, an emerging body of research has been conducted to identify the influences of prosocial video games. These studies found that playing prosocial video games helped reduce aggressive behaviors and increase prosocial behaviors. ${ }^{4,8-10}$ The general learning model $(\mathrm{GLM})^{11}$ has been widely used to explain these effects. ${ }^{8,12,13} \mathrm{It}$ has been proposed that situational variables (e.g., video games) are related to internal states, including cognition, affect and arousal, and a change in internal states could in turn influence behavioral outcomes. ${ }^{14,15}$

Even though research on the GLM showed that video games affect cognition, it has not explained how this change occurs. The social information processing theory ${ }^{16,17}$ may help to understand the potential mechanism. It proposed how individuals interact with and respond to social information, and pointed out that when facing social information, individuals first attend to and encode cues, then represent and interpret the inputs before selecting behavioral responses. As the first and critical step in the generation of behavior, attention is a crucial function that combines sensation and the perception of the original information, and further influences the following steps of information processing. ${ }^{16}$ Thus, attentional bias to game content might be the key to understand the influences of video games on cognition.

The influences of violent video games on attentional bias toward aggressive information has been investigated in previous studies. ${ }^{18-22}$ In terms of prosocial video games, Greitemeyer and Ossward ${ }^{12}$ proposed that the attentional bias to prosocial information might be the reason why players reduce aggressive behavior after game exposure. However, the link between prosocial video game exposure and attentional bias to prosocial information has not been directly investigated.

Previous studies ${ }^{23,24}$ used the cueing paradigm to measure attentional bias. For example, Fox et al. ${ }^{23}$ employed this paradigm to investigate the attentional bias for threat and found that high trait anxious people showed attention disengagement to

\footnotetext{
${ }^{1}$ Key Laboratory of Brain, Cognition and Education Sciences, South China Normal University, Ministry of Education, Guangzhou, P.R. China.

${ }^{2}$ School of Psychology, Center for Studies of Psychological Application, and Guangdong Key Laboratory of Mental Health and Cognitive Science, South China Normal University, Guangzhou, P.R. China.

${ }^{3}$ Laboratory for Behavioral and Regional Finance, Guangdong University of Finance, Guangzhou, P.R. China.
} 
threat words. They explained that threat-related stimuli might have a biological basis for being prioritized by the attentional system. Therefore, threat-related stimuli can more easily cause autonomic responses and hold attention, which in turn result in reaction time (RT) difference between threat-related stimuli and control stimuli in the visual attention tasks. Another similar study ${ }^{24}$ revealed that the attentional bias might vary depending on the duration of stimuli presentation. Accordingly, we propose that the priority of attentional processing of prosocial information may cause autonomic responses and in turn result in different RTs to prosocial and neutral stimuli in visual attention tasks and this effect is likely to vary by the duration of stimuli presentation.

The aim of this study, therefore, was to investigate different components (i.e., the orientation component and the disengage component) of attentional bias toward prosocial information after short-term prosocial video game exposure, at different presentation durations (100, 500, 1,250 milliseconds [ms]). We chose to examine these two components because the primary adaptive function of attention is to facilitate fast and accurate perception of the environment while maintaining resources to process stimulus inputs. ${ }^{23,25}$ Particularly, we were interested in the time course of attentional bias. According to previous research related to food ${ }^{26}$ and threat cues, ${ }^{27}$ which suggested that attentional bias occurred at $100 \mathrm{~ms}$, we hypothesized that attentional bias toward prosocial stimuli might also occur at the early stage of the cognitive process.

\section{Materials and Methods}

\section{Participants}

Sixty undergraduate students were recruited to participate in the study (all males, mean age 20.26 years, range 19-23 years). Half of the participants were randomly assigned to the prosocial game group and the others to the neutral game group. All participants had normal or corrected-to-normal vision. Written informed consent was obtained from each participant before experiments. All materials and procedures were approved by the Human Research Ethics Committee, South China Normal University.

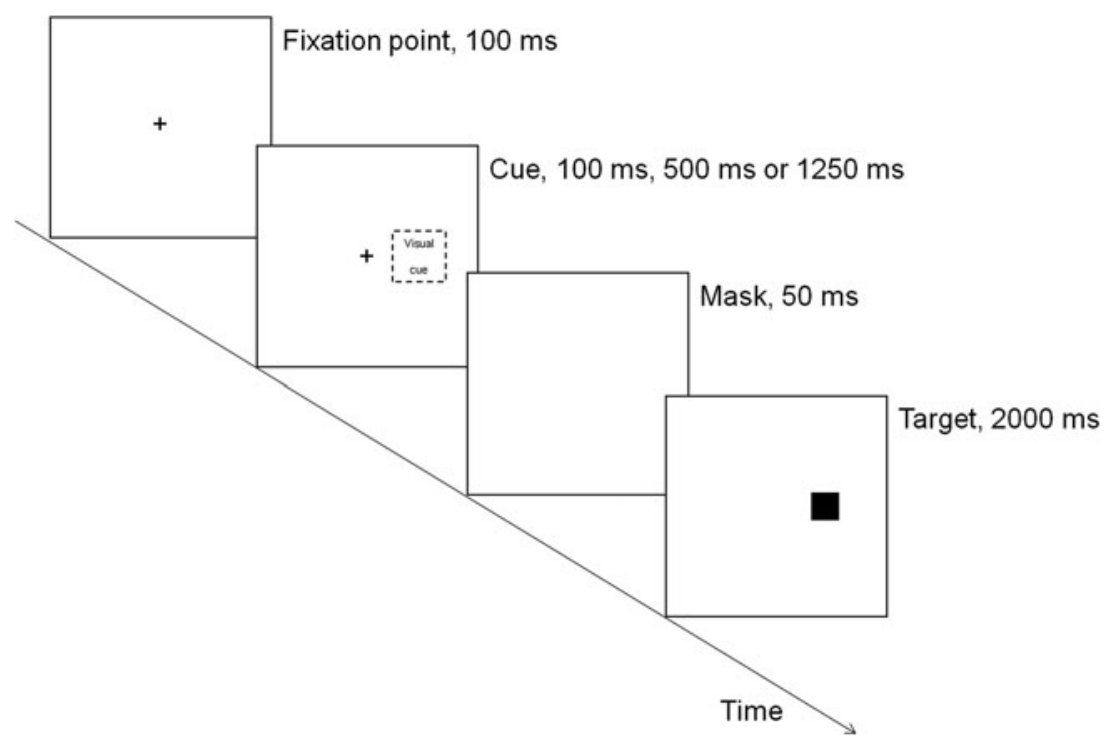

\section{Measures}

Video games. According to previous studies, ${ }^{9,12,14,28}$ Pure Pinball was used as the neutral video game and Lemmings $I$ as the prosocial game in this study. The task in Pure Pinball was to keep a pinball on the desk by flapping two baffles and the task in Lemmings I was to guide and protect a troop of lost lemmings under several difficulties caused by the terrain with the goal to arrive home.

When finishing the game, participants were required to rate the game by a postexperimental questionnaire. 9,29 Participants were asked to rate several dimensions of the game, including action-packed, enjoyable, exciting, entertaining, fun, involving, hard to play, frustrating, ability on the game, violent content, and prosocial content, using 10 -point scales. For example, participants rated their agreement on the prosocial content with the statement that "The game involves helping behavior" using a 10-point scale $(0=$ totally disagree, $9=$ totally agree $)$ for the prosocial content item of the questionnaire.

Spatial cueing paradigm. A spatial cueing paradigm (adapted from Fox et al. ${ }^{23}$ ) was used to detect attentional bias. In this paradigm, a fixation point was first presented at the center of the computer screen for $1,000 \mathrm{~ms}$. A visual cue (an image, either prosocial or neutral) was then presented on one side of the screen for a variable amount of time (100, 500 or $1,250 \mathrm{~ms}$, balanced on cue validity and cue image). A mask was then presented for $50 \mathrm{~ms}$, followed by the target stimulus (a solid black square) on one side of the screen (either the same side or the opposite side of the cue) for 2,000 ms (Fig. 1). Participants were seated about $50 \mathrm{~cm}$ from the computer monitor and asked to indicate the horizontal position of the target stimulus as quickly and as accurately as possible by pressing the " $F$ " button for the left side and " $\mathrm{J}$ ", for the right. Participants practiced on 20 trials and received 144 target trials. The target trials included 72 trials with a prosocial cue and 72 trials with a neutral cue. Two-thirds of the cues were valid (target stimulus presented on the same side of the cue) and the others were invalid (target stimulus presented on the opposite side of the cue).
FIG. 1. Procedure of the spatial cueing paradigm. In a single trial of the spatial cueing task, a fixation point was first presented in the middle of the computer screen for $1,000 \mathrm{~ms}$. A visual cue (an image, either prosocial or neutral) was then presented on one side of the screen for a variable amount of time $(100,500$, or $1,250 \mathrm{~ms}$ ), followed by the presentation of a target stimulus (a black solid square) on one side of the screen (either the same side of the cue or the oppose side) for 2,000 ms. ms, milliseconds. 
In the spatial cueing paradigm, when the cue and the target are presented on the same side of the screen (i.e., valid cue), the cue image guides attention orientation when participants respond to the target. ${ }^{23,30}$ When the cue and the target are presented on opposite sides of the screen (i.e., invalid cue), participants have to disengage their attention from the cue image and orient to the target. ${ }^{23,31}$ By manipulating cue validity, we can distinguish different components (attention orientation and attention disengagement) of attentional bias. ${ }^{23}$

Images for the spatial cueing paradigm were assessed in a pilot study. Two hundred cue images were collected from open access websites and edited into $220 \times 220$ pixels with the same color saturation and brightness. These images were assessed by 20 male undergraduates (mean age 21.3 years, range 19-23 years), and a 9-point scale was used to rate their agreement with a statement saying "The image involves prosocial content." Students who took part in the assessment were not selected to participate in the experiment. Finally, 72 prosocial cue images and 72 neutral cue images were selected according to the ratings. The selected prosocial cue images contained more prosocial content than the neutral cue images, $t(142)=-123.05, p<0.001$, Cohen's $d=20.52$.

\section{Procedure}

Participants were randomly assigned to the prosocial game group or the neutral game group when they arrived, and then were informed of the goal of the game. Participants in the prosocial game group played Lemmings I for 30 minutes, whereas those in the neutral group played Pure Pinball for the same amount of time. All the participants were required to complete a postexperimental questionnaire after the game. Afterward, participants in both groups practiced the spatial cueing task and performed the formal task.

\section{Statistical analysis}

A 2 (game type: prosocial or neutral) $\times 2$ (cue validity: valid or invalid) $\times 2$ (cue image: prosocial or neutral) $\times 3$ (cue presentation time: 100, 500, and 1,250 ms) design was applied. Game type was a between-subjects factor, whereas cue validity, cue image, and presentation time were within-subject factors.

Trials with an incorrect response or with RT that exceeded two standard deviations from the grand mean were excluded from further analysis. Excluded data accounted for 1.32 percent of the overall data $(0.53$ percent from the prosocial game group and 0.79 percent from the neutral group). We first conducted a four-factor mixed-design analysis of variance (ANOVA) with RT as the dependent variable. To further examine the performance of specific cue presentation time and to test our prediction that attentional bias would occur at the early stage but not at other durations, we conducted 2 (game type: prosocial or neutral) $\times 2$ (cue validity: valid or invalid) $\times 2$ (cue image: prosocial or neutral) mixeddesign ANOVA for each cue presentation time (i.e., 100, 500 , and $1,250 \mathrm{~ms}$ ) separately.

\section{Results}

The rating scores

of the postexperimental questionnaire

Rating scores showed that Lemmings I contained more prosocial content than Pure Pinball, $t(58)=5.36, p<0.001$,
Table 1. Descriptive Statistics for All Variables Under 100 ms Cue Presentation Time ( $M \pm S D$ Ms)

\begin{tabular}{llcc}
\hline $\begin{array}{l}\text { Cue } \\
\text { validity }\end{array}$ & $\begin{array}{c}\text { Cue } \\
\text { image }\end{array}$ & $\begin{array}{c}\text { Prosocial } \\
\text { game group }\end{array}$ & $\begin{array}{c}\text { Neutral } \\
\text { game group }\end{array}$ \\
\hline Valid & Neutral & $343.90 \pm 35.20$ & $333.16 \pm 34.84$ \\
& Prosocial & $334.11 \pm 36.61$ & $331.21 \pm 36.17$ \\
Invalid & Neutral & $334.44 \pm 37.44$ & $332.12 \pm 42.13$ \\
& Prosocial & $343.05 \pm 37.49$ & $334.15 \pm 38.87$ \\
\hline
\end{tabular}

$S D$, standard deviation; ms, milliseconds.

Cohen's $d=1.388$, but no other significant difference was found. These results suggested that the experimental treatment is effective, the two games do convey different contents and the other dimensions do not significantly influence the game experience of the participants. To further confirm that these dimensions had no significant effects on the participants' task performance, we added the rating scores as covariates in the ANOVA analysis and results showed that none of these dimensions have a significant between-subject effect on participants' RTs in the task, $F \mathrm{~s}<0.985, p \mathrm{~s}>0.32$.

\section{Four-factor ANOVA}

Descriptive statistics for each condition are shown in Tables 1-3. The main effect of cue presentation time was significant in the four-factor ANOVA, $F(2,57)=73.32$, $p<0.001, \eta^{2}=0.727$. Post hoc comparisons using $t$ test with Bonferroni correction indicated that mean RT of the $1,250 \mathrm{~ms}$ condition (313.14 ms) was significantly shorter than both the $100 \mathrm{~ms}$ condition $(335.77 \mathrm{~ms})$ and the $500 \mathrm{~ms}$ condition $(332.78 \mathrm{~ms})$, whereas the $100 \mathrm{~ms}$ condition and the $500 \mathrm{~ms}$ condition did not significantly differ from each other. Moreover, the interaction among cue presentation time, cue validity, and cue image was found to be significant, $F(2,57)=3.90$, $p=0.026, \eta^{2}=0.124$, whereas no other significant main effects or interactions were found. Post hoc tests indicated that the mean RTs for each of the combinations of the conditions (cue validity $\times$ cue image) under $1,250 \mathrm{~ms}$ condition was significantly shorter than the combinations of the conditions under 100 and $500 \mathrm{~ms}$ conditions with the mean RT differences ranging from 15.66 to $27.49 \mathrm{~ms}$, no other significant post hoc results were found. These results indicate that participants respond significantly faster when the stimuli presented for 1,250 ms compared with other duration conditions. To test whether the attention bias would only occur at the early stage (i.e., $100 \mathrm{~ms}$ presentation time) as previously mentioned, we further performed a three-factor ANOVA for each presentation time.

Table 2. Descriptive Statistics for All Variables Under 500 ms Cue Presentation Time ( $M \pm S D$ ms)

\begin{tabular}{llcc}
\hline $\begin{array}{l}\text { Cue } \\
\text { validity }\end{array}$ & $\begin{array}{c}\text { Cue } \\
\text { image }\end{array}$ & $\begin{array}{c}\text { Prosocial } \\
\text { game group }\end{array}$ & $\begin{array}{c}\text { Neutral } \\
\text { game group }\end{array}$ \\
\hline Valid & Neutral & $340.47 \pm 37.36$ & $331.49 \pm 39.20$ \\
& Prosocial & $340.83 \pm 32.96$ & $327.61 \pm 33.11$ \\
Invalid & Neutral & $330.92 \pm 31.73$ & $331.07 \pm 38.56$ \\
& Prosocial & $335.10 \pm 36.21$ & $324.71 \pm 33.62$ \\
\end{tabular}


Table 3. Descriptive Statistics for All Variables Under 1,250 ms Cue Presentation Time ( $M \pm S D$ ms)

\begin{tabular}{llcc}
\hline $\begin{array}{l}\text { Cue } \\
\text { validity }\end{array}$ & $\begin{array}{c}\text { Cue } \\
\text { image }\end{array}$ & $\begin{array}{c}\text { Prosocial } \\
\text { game group }\end{array}$ & $\begin{array}{c}\text { Neutral } \\
\text { game group }\end{array}$ \\
\hline Valid & Neutral & $317.75 \pm 36.14$ & $310.44 \pm 35.03$ \\
& Prosocial & $316.63 \pm 35.22$ & $309.56 \pm 30.98$ \\
Invalid & Neutral & $315.55 \pm 33.40$ & $312.94 \pm 36.93$ \\
& Prosocial & $313.10 \pm 30.74$ & $309.12 \pm 34.24$ \\
\hline
\end{tabular}

\section{0 ms condition}

Descriptive statistics for all variables under $100 \mathrm{~ms}$ cue presentation time are shown in Table 1. No main effect was significant in this condition, $F \mathrm{~s}<0.457, p \mathrm{~s}>0.502$. However, a significant interaction between cue validity and cue image was found, $F(1,58)=10.46, p=0.002, \eta^{2}=0.157$. Simple effect analysis showed that the mean RT in response to prosocial images $(332.66 \mathrm{~ms})$ was shorter than the mean RT in response to neutral images $(338.53 \mathrm{~ms})$ when the cue was valid, $F(1,58)=10.08, p=0.002, \eta^{2}=0.148$, whereas there was no significant difference between RTs for prosocial and neutral images when the cue was invalid.

Importantly, the interaction among cue validity, cue image, and game group was also significant, $F(1,58)=4.36$, $p=0.041, \eta^{2}=0.072$. To break down this interaction, we examined the data for each game group separately. Results showed that, in the prosocial game group, the mean RT in response to prosocial images $(334.11 \mathrm{~ms})$ was shorter than the mean RT in response to neutral images $(343.90 \mathrm{~ms})$ when the cue was valid, $F(1,58)=14.03, p<0.001, \eta^{2}=0.195$, whereas the mean RT for prosocial images $(343.05 \mathrm{~ms})$ was longer than for neutral images $(334.44 \mathrm{~ms})$ when the cue was invalid, $F(1,58)=4.64, p=0.036, \eta^{2}=0.074$ (Fig. 2a). No significant simple effect was found in the neutral game group (Fig. 2b).

\section{0 ms condition}

Descriptive statistics for all variables under $500 \mathrm{~ms}$ cue presentation are shown in Table 2. Again, no main effects

\section{a Prosocial game group}

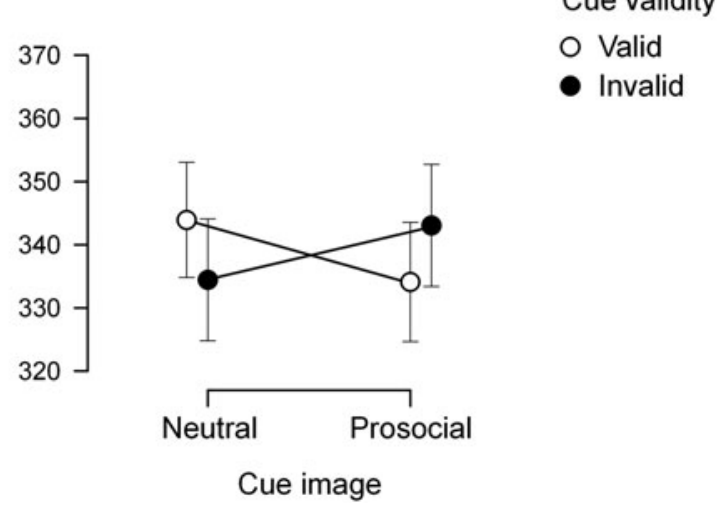

were significant in this condition, $F \mathrm{~s}<3.754, p s>0.058$. However, a significant interaction between game group and cue image was found, $F(1,58)=4.12, p=0.047, \eta^{2}=0.069$. Simple effects analysis revealed that difference in mean RT in response to the prosocial images $(337.94 \mathrm{~ms})$ and neutral images $(335.70 \mathrm{~ms})$ was not significant in either the prosocial game group, $F(1,58)=0.778, p=0.381$ or the neutral game group, $F(1,58)=3.957, p=0.052$.

\section{1,250 ms condition}

Descriptive statistics for all variables under $1,250 \mathrm{~ms}$ cue presentation are shown in Table 3 . No significant main effects or interactions were found in this condition, $F \mathrm{~s}<1.540$, $p s>0.22$.

\section{Discussion}

In this study, we investigated whether short-term exposure to prosocial video games induced attentional bias to prosocial stimuli. The current results revealed that both orientation and disengagement subcomponents of attentional bias existed, but the effects only occurred at the early stage of attention processing.

Specifically, in the $100 \mathrm{~ms}$ condition, RT facilitation was found in the prosocial video game group on valid trials with prosocial cues, compared with those with neutral cues, indicating an attention orientation. Furthermore, RT delay with prosocial cues also emerged in the prosocial game group when the cue was invalid, whereas no significant RT delay was found for neutral cues, indicating the difficulty of attention disengagement. However, this effect was not found in the $500 \mathrm{~ms}$ and the $1,250 \mathrm{~ms}$ conditions.

The findings in $100 \mathrm{~ms}$ condition were consistent with previous research on violent video games. Previous studies found that violent video game players showed attentional bias toward aggressive information at the early stages (e.g., $129.42 \mathrm{~ms}^{18}$ and $100 \mathrm{~ms}^{19}$ ) of attention processing and revealed that the attentional bias might be spontaneous and related to video game experience. Likewise, we found both attention orientation and difficulty in attention disengagement

\section{b Neutral game group}
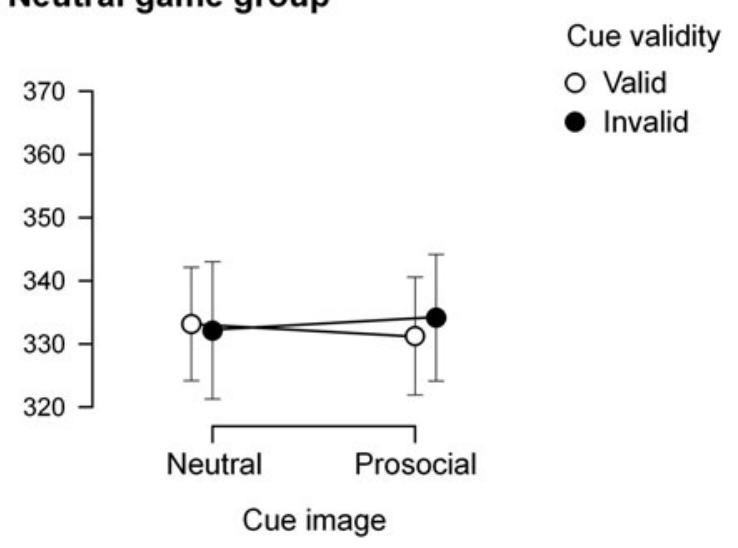

FIG. 2. Results of the simple effect analysis of the interaction among cue validity, cue image, and game group under $100 \mathrm{~ms}$ cue presentation time. (a) Displayed the results of the prosocial video game group and (b) displayed the results of the neutral video game group. Lines connecting hollow dots represented valid cues and lines connecting solid dots represented invalid cues. The y-axis showed the reaction times, and the $\mathrm{x}$-axis showed cue image types (i.e., neutral and prosocial). Error bars displayed the standard errors. 
toward prosocial information at the early stage of attention processing after prosocial video game exposure.

The prosocial game used in this study contained protecting and helping behaviors. It might be that the game content activated physiological constructs such as the parasympathetic nervous system and the hypothalamic-pituitary-adrenal axis ${ }^{32,33}$ and inspired relevant cognitive schema, ${ }^{34}$ which in turn increases the accessibility of prosocial information. Therefore, in trials with valid cues participants might attend to the target stimuli (on the same side of the prosocial cue) with only a small amount of cognitive resources compared with those with neutral cues, thus reflecting in shorter RTs in these trials (attention orientation). Likewise, participants had to consume more cognitive resources to disengage their attention of the prosocial cues and attend to the target stimuli when the cue is invalid, reflecting in longer RTs in trials with invalid prosocial cues compared with those with invalid neutral cues (attention disengagement). ${ }^{18,35}$

However, this effect was not observed as the processing time became longer (i.e., 500 or 1,250 ms). We speculate that when there is enough time for processing, the control ability of cognitive functions takes the lead in the response instead of the spontaneous attentional bias.

In summary, the results provide preliminary evidence of the influence of prosocial video games on cognitive processing and may potentially contribute to the game rating systems. However, it should be noted that only three presentation times were selected in this study, attentional bias under other time windows remains unclear. Furthermore, only one visual task was used to test attentional bias and this might cause a task impurity problem. Given these limitations of this study, future research may use paradigms in other modalities (e.g., audio tasks and written tasks) to further explore attentional bias after prosocial video game exposure with different cue presentation time.

\section{Author Disclosure Statement}

No competing financial interests exist.

\section{Funding Information}

This study was funded by the National Natural Science Foundation of China (31671154 and 31600901).

\section{References}

1. Gentile D. Pathological video-game use among youth ages 8 to 18: a national study. Psychological Science 2009; 20: 594-602.

2. Tear MJ, Nielsen M. Video games and prosocial behavior: a study of the effects of non-violent, violent and ultraviolent gameplay. Computers in Human Behavior 2014; 41: 8-13.

3. Dill KE, Gentile DA, Richter WA, et al. (2005) Violence, sex, race, and age in popular video games: a content analysis. In Cole E, Daniel JH, eds. Psychology of women book series. Featuring females: feminist analyses of media. Washington, DC: American Psychological Association, pp. $115-130$.

4. Greitemeyer T, Mügge DO. Video games do affect social outcomes: a meta-analytic review of the effects of violent and prosocial video game play. Personality and Social Psychology Bulletin 2014; 40:578-589.
5. Diaz RL, Wong U, Hodgins DC, et al. Violent video game players and non-players differ on facial emotion recognition. Aggressive Behavior 2016; 42:16-28.

6. Gentile DA, Bender PK, Anderson CA. Violent video game effects on salivary cortisol, arousal, and aggressive thoughts in children. Computers in Human Behavior 2017; 70:39-43.

7. You S, Kim E, No U. Impact of violent video games on the social behaviors of adolescents: the mediating role of emotional competence. School Psychology International 2014; 36:94-111.

8. Greitemeyer T. Effects of prosocial media on social behavior when and why does media exposure affect helping and aggression? Current Directions in Psychological Science $2011 ; 20: 251-255$.

9. Saleem M, Anderson CA, Gentile DA. Effects of prosocial, neutral, and violent video games on children's helpful and hurtful behaviors. Aggressive Behavior 2012; 38:281-287.

10. Harrington B. (2016) Video game use and prosocial behaviour: an investigation into the relationship between prosocial video game use, empathy and prosocial behaviour in children and adolescents from different socio-economic groups (master's thesis). http://hdl.handle.net/10197/8536 (accessed May 10, 2018).

11. Buckley KE, Anderson CA. (2006) A theoretical model of the effects and consequences of playing video games. In Vorderer P, Bryant J, eds. Playing video games-motives, responses, and consequences. Mahwah, NJ: LEA, pp. 363378.

12. Greitemeyer T, Osswald S. Prosocial video games reduce aggressive cognitions. Journal of Experimental Social Psychology 2009; 45:896-900.

13. Gentile DA, Groves C, Gentile JR. (2014) The general learning model: Unveiling the learning potential from video games. New York: Oxford University Press.

14. Greitemeyer T, Osswald S. Effects of prosocial video games on prosocial behavior. Journal of Personality and Social Psychology 2010; 98:211-221.

15. Liu Y, Teng Z, Lan H, et al. Short-term effects of prosocial video games on aggression: an event-related potential study. Frontiers in Behavioral Neuroscience 2015; 9:193.

16. Crick NR, Dodge KA. A review and reformulation of social information-processing mechanisms in children's social adjustment. Psychological Bulletin 1994; 115:74-101.

17. Crick NR. Social information-processing mechanisms in reactive and proactive aggression. Child Development 1996; 67:993.

18. Zhao C, Gao X, Zhou Q, et al. Violent video game players' attentional bias toward aggressive pictures: evidence from ERPs. Studies of Psychology and Behavior 2016; 14:584590.

19. Zhen SJ, Xie XD, Hu LP, et al. Study on the mechanism of violent games on individual attention bias. Journal of South China Normal University: Social Science Edition 2013; 2: 67-73.

20. Kirsh SJ, Olczak PV, Mounts JRW. Violent video games induce an affect processing bias. Media Psychology 2005; 7:239-250.

21. Ding DQ, Yan WU. Research on attention bias to aggression-related cues in violent video-game users. Journal of Educational Science of Hunan Normal University 2014; 5:115-118. 
22. Liu Y, Lan H, Teng Z, et al. Facilitation or disengagement? Attention bias in facial affect processing after short-term violent video game exposure. PLoS One 2017; 12:e0172940.

23. Fox E, Russo R, Bowles R, et al. Do threatening stimuli draw or hold visual attention in subclinical anxiety? Journal of Experimental Psychology General 2001; 130:681-700.

24. Koster EH, Verschuere B, Crombez G, et al. Time-course of attention for threatening pictures in high and low trait anxiety. Behaviour Research and Therapy 2005; 43:1087-1098.

25. La BD. (1995) Attentional processing: the brain's art of mindfulness. Cambridge, MA: Harvard University Press.

26. Deluchi M, Costa FS, Rogério F, et al. Attentional bias to unhealthy food in individuals with severe obesity and binge eating. Appetite 2017; 108:471-476.

27. Cooper RM, Langton SRH. Attentional bias to angry faces using the dot-probe task? It depends when you look for it. Behaviour Research and Therapy 2006; 44:1321-1329.

28. Greitemeyer T, Osswald S, Brauer M. Playing prosocial video games increases empathy and decreases schadenfreude. Emotion 2010; 10:796-802.

29. Carnagey NL, Anderson CA, Bushman BJ. The effect of video game violence on physiological desensitization to real-life violence. Journal of Experimental Social Psychology 2007; 43:489-496.

30. Marzouki Y, Grainger J, Theeuwes J. Exogenous spatial cueing modulates subliminal masked priming. Acta Psychologica 2007; 126:34-45.

31. Yiend J, Mathews A. Anxiety and attention to threatening pictures. The Quarterly Journal of Experimental Psychology $2001 ; 54: 665-681$.
32. Miller JG. Physiological mechanisms of prosociality. Current Opinion in Psychology 2018; 20:50-54.

33. Alink LRA, Cicchetti D, Kim J, et al. Longitudinal associations among child maltreatment, social functioning, and cortisol regulation. Developmental Psychology 2012; 48: 224-236.

34. Carnagey NL, Anderson CA. The effects of reward and punishment in violent video games on aggressive affect, cognition, and behavior. Psychological Science 2005; 16: 882-889.

35. Koster EH, Crombez G, Verschuere B, et al. Selective attention to threat in the dot probe paradigm: differentiating vigilance and difficulty to disengage. Behaviour Research and Therapy 2004; 42:1183-1192.

Address correspondence to: Dr. Shuangju Zhen School of Psychology Center for Studies of Psychological Application and Guangdong Key Laboratory of Mental Health and Cognitive Science South China Normal University No.55, West Zhongshan Avenue Tianhe

Guangzhou 510631 P.R. China

E-mail: shuangjuzhen@foxmail.com 